九州大学学術情報リポジトリ

Kyushu University Institutional Repository

\title{
ON DECOMPOSABILITY OF PROBABIKITY MEASURES ON A SEPARABLE METRIC SPACE ACTED UPON BY A COMPACT METRIC GROUP
}

Nagai, Takeaki

Faculty of Science, Kagoshima University

https://doi.org/10.5109/13036

出版情報 : 統計数理研究. 13 (3/4), pp. 57-63，1969-03. Research Association of Statistical Sciences

バージョン：

権利関係 : 


\title{
ON DECOMPOSABILITY OF PROBABILITY MEASURES ON A SEPARABLE METRIC SPACE ACTED UPON BY A COMPACT METRIC GROUP
}

\author{
By
}

\author{
Takeaki NAGAI*
}

(Received Jan. 5, 1969)

\section{$\S 1$. Summary.}

In this paper we consider probability measures on a complete separable metric space $T$ (or on a topological subspace $T$ of a complete separable metric space $T^{*}$ ) with which a compact metric group $G$ of homeomorphisms acting on $T$ is associated.

Let $\mu$ be an arbitrary probability measure on $T$ invariant under every $g \in G$. Then it is shown that for an arbitrary small $\varepsilon>0$, there always exists a compact set $\Lambda_{0} \subset T$ and a probability measure $\mu_{0}$ on $T$ invariant under every $g \in G$ such that

(i) $G \cdot \Lambda_{0}=\Lambda_{0}$.

(ii) The support of $\mu_{0}$ is a closed subset of $\Lambda_{0}$.

(iii) For every Borel set $A,\left|\mu(A)-\mu_{0}(A)\right|<\varepsilon$.

(iv) Let $V$ be a Borel set in $\Lambda_{0}$ such that $G \cdot V=\Lambda_{0}$ and $G v_{1} \cap G v_{2}=\phi$ if $v_{1} \neq v_{2}$, $\left(v_{1}, v_{2} \in V\right)$, whose existence is shown in lemma 3 . Then the probability measure $\mu_{0}$ is decomposed into a direct product measure of the normalized Haar measure of $G$ and a probability measure on $V$.

\section{$\S 2$. Decomposition of $G$-invariant probability measures.}

Let $T$ be a complete separable metric space or a topological subspace of a complete separable metric space $T^{*}$. Let $G$ be a compact metric group of homeomorphisms acting on $T$ such that $G \cdot T=T$ and the mapping $(g, t) \rightarrow g t$ from $G \times T$ into $T$ is continuous.

For any metric space $X$ we shall denote by $\mathfrak{B}_{X}$ the $\sigma$-field of Borel subsets of $X$. that is,

Let $\mu$ be a probability measure defined on $\mathfrak{B}_{T}$ and invariant under every $g \in G$,

$$
\mu(T)=1 \text {. }
$$

For each $g \in G, \mu(g A)=\mu(A), A \in \mathfrak{B}_{T}$.

Lemma 1. Let $g$ and $t$ be any elements of $G$ and $T$ respectively but be fixed. Let $W$ be an arbitrary neighbourhood of $g t \in T$. Then we can always find a neighbourhood $U$ of $t$ such that $W=g U$.

\footnotetext{
* Faculty of Science, Kagoshima University, Kagoshima.
} 
Proof. $g: t \rightarrow g t$ is a homeomorphism from $T$ onto itself and $g^{-1}(W)=g^{-1} \cdot W$ is an inverse image of the open set $W$ under the mapping $g$. Hence, $g^{-1} \cdot W$ is open.

Since $t=g^{-1} \cdot g t \in g^{-1} W, g^{-1} W$ is a neighbourhood of $t$. Thus, by writing $U=g^{-1} \cdot W$, we see that $W=g U$.

Lemma 2. Let $\Lambda$ be the support of the probability measure $\mu$.

Then $G \cdot \Lambda=\Lambda$.

Proof. $A$ is a set of all elements $t \in T$ such that for any neighbourhood $U$ of $t$, $\mu(U)>0$. (See [2], page 28).

Let $W$ be an arbitrary neighbourhood of $g t \in T$, where $g$ and $t$ are any fixed elements of $G$ and $\Lambda$ respectively.

Then from lemma 1 , we can choose a neighbourhood $U$ of $t$ such that $W=g U$. Thus, we see that $\mu(W)=\mu(g U)=\mu(U)>0$. This implies that $g t \in \Lambda$ for all $g \in G$ and hence $G \cdot \Lambda \subset \Lambda$.

Since $\Lambda \subset G \cdot \Lambda$, we have proved that $G \cdot \Lambda=\Lambda$.

Lemma 3. Let $\Gamma \subset T$ be a compact set invariant under every $g \in G$.

Then there is a Borel set $V \subset T$ such that

$$
\begin{aligned}
& \Gamma=G \cdot V, \\
& G v_{1} \cap G v_{2}=\phi \quad \text { if } \quad v_{1} \neq v_{2}, \quad\left(v_{1}, v_{2} \in V\right) .
\end{aligned}
$$

Proof. For any two points $t_{1}, t_{2} \in \Gamma$, we shall say that $t_{1} \sim t_{2}$ if there exists $g \in G$ such that $g t_{1}=t_{2}$. " $\sim$ " is an equivalence relation. Let $M$ be the space of all such equivalence classes. Let $[t]$ denote the equivalence class containing $t$. Then, since the mapping $t \rightarrow[t]$ from $\Gamma$ into $M$ is continuous under the quotient topology and is onto, we see that $M$ is a continuous image of the compact set $\Gamma$ under this mapping and hence $M$ is a compact metric space.

Thus, by a theorem of Federer and Morse (see [2], page 23, Theorem 4.2), it follows that there exists a Borel set $V \subset \Gamma$ satisfying (3) and (4).

Now, we have the following:

Lemma 4. Let $\Gamma \subset T$ be the set considered in lemma 3 and suppose for any $g \in G$, $g \neq e$, there is no fixed point in $\Gamma$.

Then the mapping

$$
\xi:(g, v) \rightarrow g v
$$

is a Borel isomorphism between $G \times V$ and $\Gamma$.

Proof. From lemma 3, we see that the mapping $\xi: G \times V \rightarrow \Gamma$ is onto. Since there is no fixed point in $\Gamma$ for any $g \in G, g \neq e, \xi$ is one-one. From our assumption, it is clear that the mapping $\xi$ is continuous.

In particular, $\xi$ is measurable. Hence, by a theorem of Kuratowski (See [2], page 21 , theorem 3.9 ), $\xi^{-1}$ is measurable.

Thus, we have proved lemma 4.

Now we have the following theorem.

Theorem 1. Let the support $A$ of $\mu$ be compact. Let $V \subset T$ be a Borel set such that $G \cdot V=\Lambda$ and $G v_{1} \cap G v_{2}=\phi$ if $v_{1} \neq v_{2},\left(v_{1}, v_{2} \in V\right)$ and the mapping $\xi:(g, v) \rightarrow g v$ be a Borel isomorphism between $G \times V$ and $\Lambda$. 
Let $C$ be $a$ Borel set in $T$ and $A$ and $B$ Borel subsets in $G$ and $V$ respectively such that

Then,

$$
\xi(A \times B)=C \cap A \in \mathfrak{B}_{A} .
$$

$$
\mu(C)=\nu(A) \cdot \rho(B),
$$

where $\nu$ is the normalized Haar measure on $G$ and $\rho$ a probability measure on $\mathfrak{B}_{V}$.

Proof. Let us notice that $A$ is a compact set in $T$ and hence $A \in \mathfrak{B}_{T}$. Therefore $\mathfrak{B}_{A}$ is the $\sigma$-field of subsets of $\Lambda$ of the form $A \cap A, A \in \mathfrak{B}_{T}$, that is, $\mathfrak{B}_{A}=\{\tilde{A}=A \cap A$ $\left.\mid A \subseteq \mathfrak{B}_{T}\right\}$.

Now let us define a set function $\tilde{\mu}$ on $\mathfrak{B}_{A}$ in such a way that:

For each $\tilde{A}=A \cap \Lambda \in \mathfrak{B}_{A}, A \in \mathfrak{B}_{T}$,

$$
\tilde{\mu}(\tilde{A})=\mu(A) .
$$

It is easy to see that $\tilde{\mu}$ is a probability measure on $\mathfrak{B}_{A}$. Indeed, for any $\tilde{A} \in \mathfrak{B}_{A}$, $0 \leqq \tilde{\mu}(\tilde{A})=\mu(A) \leqq 1$ and $\tilde{\mu}(\Lambda)=\mu(\Lambda)=1$.

Let $\left\{\tilde{A}_{\nu}=A_{\nu} \cap \Lambda, A_{\nu} \in \mathfrak{B}_{T}, \nu=1,2, \cdots\right\}$ be a sequence of disjoint sets in $\mathfrak{B}_{A}$. However $\left\{A_{\nu} ; \nu=1,2, \cdots, A_{\nu} \in \mathfrak{B}_{T}\right\}$ are not necessarily disjoint.

Let us notice that $\bigcup_{\nu=1}^{\infty} A_{\nu}=\bigcup_{\nu=1}^{\infty}\left(A_{\nu} \cap \Lambda\right) \cup \bigcup_{\nu=1}^{\infty}\left(A_{\nu} \cap \Lambda^{c}\right)$, and $\bigcup_{\nu=1}^{\infty}\left(A_{\nu} \cap \Lambda\right)$ and $\bigcup_{\nu=1}^{\infty}\left(A_{\nu} \cap \Lambda^{c}\right)$ are disjoint. Since $\bigcup_{\nu=1}^{\infty} \tilde{A}_{\nu}=\bigcup_{\nu=1}^{\infty} A_{\nu} \cap A$, we have

$$
\begin{aligned}
\tilde{\mu}\left(\bigcup_{\nu=1}^{\infty} \tilde{A}_{\nu}\right) & =\mu\left(\bigcup_{\nu=1}^{\infty} A_{\nu}\right) \\
& =\mu\left(\bigcup_{\nu=1}^{\infty}\left(A_{\nu} \cap A\right)\right)+\mu\left(\bigcup_{\nu=1}^{\infty}\left(A_{\nu} \cap \Lambda^{c}\right)\right) .
\end{aligned}
$$

From the fact that $\mu\left(\bigcup_{\nu=1}^{\infty}\left(A_{\nu} \cap \Lambda^{c}\right)\right) \leqq \mu\left(\Lambda^{c}\right)=0$, it follows that

$$
\begin{aligned}
\tilde{\mu}\left(\bigcup_{\nu=1}^{\infty} \tilde{A}_{\nu}\right) & =\mu\left(\bigcup_{\nu=1}^{\infty}\left(A_{\nu} \cap A\right)\right) \\
& =\sum_{\nu=1}^{\infty} \mu\left(A_{\nu} \cap A\right) \\
& =\sum_{\nu=1}^{\infty} \tilde{\mu}\left(\tilde{A}_{\nu}\right) .
\end{aligned}
$$

Thus, we have proved that $\tilde{\mu}$ is a probability measure on $\mathfrak{B}_{A}$.

By $\mu^{*}$ let us denote the probability measure on $\mathfrak{B}_{G \times V}$ induced by the mapping $\xi^{-1}$ from $\Lambda$ onto $G \times V$, that is, $\mu^{*}$ is the probability measure such that for any $A \times B \in \mathfrak{B}_{G \times V},\left(A \in \mathfrak{B}_{G}, B \in \mathfrak{B}_{V}\right)$,

$$
\mu^{*}(A \times B)=\tilde{\mu}(\xi(A \times B)) .
$$

Since both $G \times V$ and $G$ are complete separable metric spaces, these are automatically separable standard Borel spaces (see [2], page 133).

Let us consider the mapping $\pi:(g, v) \rightarrow g$ from $G \times V$ onto $G$. Then it is clear that $\pi$ is measurable, since for any $E \in \mathfrak{B}_{G}, \pi^{-1}(E)=E \times V \in \mathfrak{B}_{G \times V}$.

Thus, it follows that there exists a regular conditional probability distribution 
of $\mu^{*}$ given $\pi$, which we shall denote by $\tilde{m}_{g}(A \times B), g \in G, A \times B \in \mathfrak{B}_{G \times V}$. This satisfies the following conditions:

(i) For each $g \in G, \tilde{m}_{g}(\cdot)$ is a probability measure on $\mathfrak{B}_{G \ltimes v}$.

(ii) For each $A \times B \in \mathfrak{B}_{G \times V}$, the mapping $g \rightarrow \tilde{m}_{g}(A \times B)$ is $\mathfrak{B}_{G}$-measurable and

(iii) $\mu^{*}(A \times B)=\int_{G} \tilde{m}_{g}(A \times B) d \nu(g)$,

where $\nu(E)=\mu^{*}\left(\pi^{-1}(E)\right)=\mu^{*}(E \times V), E \in \mathfrak{B}_{G}$. (See [2], page 146).

It is obvious that $\nu$ is a probability measure on $\mathfrak{B}_{G}$.

In particular, we have for any $E \in \mathfrak{B}_{G}$,

$$
\begin{aligned}
\mu^{*}\left(A \times B \cap \pi^{-1}(E)\right) & =\mu^{*}((A \cap E) \times B) \\
& =\int_{E} \tilde{m}_{g}(A \times B) d \nu(g), \quad A \times B \in \mathfrak{B}_{G \times \boldsymbol{V}} .
\end{aligned}
$$

Let us write

$$
m_{g}(B)=\tilde{m}_{g}(G \times B), \quad B \in \mathfrak{B}_{V} .
$$

Then we have by putting $A=G$ in (6),

$$
\mu^{*}(E \times B)=\int_{E} m_{g}(B) d \nu(g),
$$

for any $E \in \mathfrak{B}_{G}$ and any $B \in \mathfrak{B}_{V}$, where $m_{g}(\cdot)$ is a probability measure on $\mathfrak{B}_{V}$.

Let $A \in \mathfrak{B}_{G}$ and $B \in \mathfrak{B}_{V}$ and $C=\left\{\eta \in T \mid \eta=g^{\prime} t, g^{\prime} \in A, t \in B\right\}$. Then for any $g \in G$,

$$
\begin{aligned}
\mu^{*}(g A \times B) & =\tilde{\mu}(\xi(g A \times B))=\tilde{\mu}(g C)=\tilde{\mu}(C) \\
& =\tilde{\mu}(\xi(A \times B))=\mu^{*}(A \times B) .
\end{aligned}
$$

Hence it follows by putting particularly $B=V$ in (7) that

$$
\nu(g A)=\mu^{*}(g A \times V)=\mu^{*}(A \times V)=\nu(A) \quad \text { for any } A \in \mathfrak{B}_{G} .
$$

This implies that $\nu$ is the normalized Haar measure on $G$.

Thus, we may write

$$
\mu^{*}(A \times B)=\int_{A} m_{g}(B) d g .
$$

Now, for any $A \in \mathfrak{B}_{G}, B \in \mathfrak{B}_{V}$ and $h \in G$,

$$
\begin{aligned}
\mu^{*}(A \times B) & =\int_{A} m_{g}(B) d g \\
& =\mu^{*}(h A \times B) \\
& =\int_{h A} m_{g}(B) d g \\
& =\int_{A} m_{h^{-1} g}(B) d g .
\end{aligned}
$$

This implies that for any $B \in \mathfrak{B}_{V}$ and any $g, g^{\prime} \in G$,

$$
m_{g}(B)=m_{g}(B) \text {, }
$$

in other words, $m_{g}(B)$ is a probability measure on $\mathfrak{B}_{V}$ independent of $g \in G$.

Let us write

$$
\rho(B)=m_{g}(B), \quad B \in \mathfrak{B}_{\boldsymbol{V}} .
$$


Then we have for any $C \in \mathfrak{B}_{T}$ such that $\xi(A \times B)=C \cap A, A \times B \in \mathfrak{B}_{\boldsymbol{G} \times \mathbf{V}}$,

$$
\begin{aligned}
\mu(C) & =\tilde{\mu}(C \cap A) \\
& =\tilde{\mu}(\xi(A \times B)) \\
& =\mu^{*}(A \times B) \\
& =\int_{A} \rho(B) d g \\
& =\nu(A) \cdot \rho(B) .
\end{aligned}
$$

Thus, we have proved theorem 1 .

Now, let us consider the case where the support of the probability measure $\mu$ is not necessarily compact in $T$.

Lemma 5. For any $\varepsilon>0$, there exists a compact set $\Lambda_{0}$ in $T$ such that

$$
\begin{aligned}
& G \cdot \Lambda_{0}=\Lambda_{0} . \\
& \mu\left(\Lambda_{0}\right)>1-\varepsilon / 3 .
\end{aligned}
$$

Proof. Every probability measure on a complete separable metric space or on a topological subspace which is a Borel subset of such a metric space is tight (See [2], page 29). Hence, for any small $\varepsilon>0$, there is a compact set $C \subset T$ such that

$$
\mu(C)>1-\varepsilon / 3 \text {. }
$$

From a theorem of Tychonoff, it follows that $G \times C$ is compact, since it is a topological product space of a compact metric group $G$ and a compact metric space $C$. From our assumption, the mapping $\gamma:(g, t) \rightarrow g t$ from $G \times T$ into $T$ is continuous and $G \cdot C=\gamma(G \times C)$ is a continuous image of a compact set $G \times C$. Hence $G \cdot C$ is compact.

Let us write $\Lambda_{0}=G \cdot C$. Then $\Lambda_{0}$ is invariant under every $g \in G$ and $\mu\left(\Lambda_{0}\right) \geqq \mu(C)$ $>1-\varepsilon / 3$.

Let us consider a probability measure $\mu_{0}$ on $\mathfrak{B}_{T}$ defined by

$$
\mu_{0}(A)=\frac{\mu\left(A \cap A_{0}\right)}{\mu\left(\Lambda_{0}\right)}, \quad A \in \mathfrak{B}_{T} .
$$

Then, since $\mu_{0}\left(A_{0}\right)=1$, the support of $\mu_{0}$ is compact.

Since $G \cdot \Lambda_{0}=\Lambda_{0}$, we see that for any $g \in G$ and $A \in \mathfrak{B}_{T}, g A \cap \Lambda_{0}=g\left(A \cap \Lambda_{0}\right)$. Thus, it is clear that for any $g \in G, \mu_{0}(g A)=\mu_{0}(A), A \in \mathfrak{B}_{T}$.

Now, we have the following theorem:

Theorem 2. For an arbitrary small $\varepsilon>0$, there exists a compact set $\Lambda_{0} \subset T$ and a probability measure $\mu_{0}$ on $\mathfrak{B}_{T}$ such that

(11) The support of $\mu_{0}$ is a closed subset of $\Lambda_{0}$.

(12) For any $g \in G$ and $A \in \mathfrak{B}_{T}$,

and

$$
\mu_{0}(g A)=\mu_{0}(A)
$$

(13) For any $A \in \mathfrak{B}_{T}$,

$$
\left|\mu(A)-\mu_{0}(A)\right|<\varepsilon .
$$

Proof. We have already shown that the existence of the compact set $\Lambda_{0}$ and the 
probability measure $\mu_{0}$ satisfying (11) and (12).

Now, we shall prove (13).

Since for any $A \subseteq \mathfrak{B}_{T}$,

$$
\mu(A)=\mu\left(A \cap A_{0}\right)+\mu\left(A \cap \Lambda_{0}^{c}\right),
$$

we have the following inequality:

$$
\begin{aligned}
\mid \mu(A) & -\mu_{0}(A) \mid \\
& =\left|\mu\left(A \cap \Lambda_{0}^{c}\right)+\mu\left(A \cap \Lambda_{0}\right)-\frac{\mu\left(A \cap \Lambda_{0}\right)}{\mu\left(\Lambda_{0}\right)}\right| \\
& \leqq \mu\left(A \cap \Lambda_{0}^{c}\right)+\mu\left(A \cap \Lambda_{0}\right) \cdot\left(1-\mu\left(\Lambda_{0}\right)\right) / \mu\left(\Lambda_{0}\right) \\
& =\mu\left(\Lambda_{0}^{c}\right) \cdot\left(1+1 / \mu\left(A_{0}\right)\right) .
\end{aligned}
$$

As long as $\varepsilon$ is not greater than $1,1+1 / \mu\left(\Lambda_{0}\right)$ is less than $5 / 2$.

Thus, we have $\left|\mu(A)-\mu_{0}(A)\right|<(5 / 6) \varepsilon<\varepsilon$, for any $A \in \mathfrak{B}_{T}$.

This completes the proof.

Thus, in generally, every probability measure on $T$ invariant under every $g \in G$ can be approximated by a direct product probability measure of the normalized Haar measure on $G$ and a probability measure on a Borel set $V$ satisfying the conditions stated in lemma 3 as closely as possible.

Example 1. Let $T=R_{2}=\{(\theta, r) \mid 0 \leqq r<\infty, 0 \leqq \theta<2 \pi\}$ and $G=S O(2)$. Let us consider a compact set $\Gamma=\{(\theta, r) \mid 0 \leqq r \leqq \alpha, 0 \leqq \theta \leqq 2 \pi\}$ and a set $V=\{(0, r) \mid 0 \leqq r \leqq \alpha\}$, where $\alpha$ is a finite real number.

Then it is obvious that $V$ satisfies the conditions (3) and (4) in lemma 3.

Let $\mu$ be a probability measure on $\mathfrak{B}_{T}$ and $\mu(\Gamma)=1$. If for any $A \in \mathfrak{B}_{\Gamma}, \mu(A)$ is proportional to the area of the set $A$, then the probability measure $\mu$ is invariant under every $g \in S O(2)$ and it is written in the view of theorem 1 as follows:

$$
\int_{A \times B} d \mu(\theta, r)=\int_{A} \frac{1}{2 \pi} d \theta \cdot \int_{B} \frac{2}{\alpha^{2}} r d r
$$

where $A \in \mathfrak{B}_{G}$ and $B \in \mathfrak{B}_{V}$.

Example 2. Let $L_{3}=\left\{(t, x, y, z) \mid t^{2}-\left(x^{2}+y^{2}+z^{2}\right)=c^{2}\right\}$, where $c$ is a real number and $(t, x, y, z)$ 's are points in 4 -dim. Euclidean space $R_{4}$. Let $G$ be a subgroup of the proper Lorentz group of the order $(3,1)$ such that its elements are of the following form :

$$
g=\left(\begin{array}{ll}
1, & 0 \\
0, & h
\end{array}\right), \quad h \in S O(3)
$$

where 0 in the above expression denotes $3 \times 1$-zero vector and also $1 \times 3$-zero vector.

Let $\mu$ be a probability measure on $L_{3}$ invariant under every $g \in G$. Let the support of the measure $\mu$ be such that

$$
\Gamma=\left\{(t, x, y, z) \in L_{3} \mid 0 \leqq x^{2}+y^{2}+z^{2} \leqq \alpha^{2}\right\},
$$

where $\alpha$ is a finite real number.

Let us consider a set

$$
V=\left\{\left(\sqrt{c^{2}+r^{2}}, r, 0,0\right) \mid 0 \leqq r \leqq \alpha\right\} \subset \Gamma .
$$


Then $V$ satisfies the conditions (3) and (4) in lemma 3.

Suppose that for any $C \in \mathfrak{B}_{\Gamma}, \mu(C)$ is proportional to the area of $C$. Then it is written in the view of theorem 1 as follows:

$$
\begin{aligned}
\int_{C} d \mu(x, y, z) & =\int_{A_{1} \times A_{2} \times B} d \mu(r, \theta, \varphi) \\
& =\int_{A_{1} \times A_{2}} \frac{1}{4 \pi} \sin \theta d \theta d \varphi \cdot \int_{B} \frac{\beta r^{2}}{\sqrt{c^{2}+r^{2}}} d r,
\end{aligned}
$$

where $C$ is a set of all points $(t, x, y, z)$ in $\Gamma$ such that

$$
\begin{aligned}
& x=r \cdot \sin \theta \cdot \cos \varphi, \\
& y=r \cdot \sin \theta \cdot \sin \varphi, \\
& z=r \cdot \cos \theta, \\
& t=\sqrt{c^{2}+r^{2}},
\end{aligned}
$$

$\langle\theta, \varphi) \in A_{1} \times A_{2} \in \mathfrak{B}_{G}$ and $r \in B \in \mathfrak{B}_{V}$, and $\beta=\left[\int_{0}^{\alpha} r^{2} /\left(c^{2}+r^{2}\right)^{1 / 2} d r\right]^{-1}$.

\section{§3. Acknowledgment.}

The author would like to express his heartiest thanks to Professor K. R. Parthasarathy and Professor J. Gani for their kind suggestions and encouragements while this paper was being prepared.

\section{References}

[1] Kelley, J. L.: “General Topology", Van Nostrand, N. Y., 1961.

[2] Parthasarathy, K. R.: “Probability measures on metric spaces," Academic Press, N. Y., 1967.

[3] Pontrjagin, L.S.: “Topological Groups," Princeton Univ. Press, Princeton, New Jersey, 1946.

[4] Prohorov, Yu. V.: Convergence of random processes and limit theorems in probability theory, Theor. Probability Appl. 1 (1956), 157-214.

[5] Simmons, G. F.: “Introdudtion to Topology and Modern Analysis," McGraw-Hill, N. Y., 1963. 